\title{
Propagation of a string across the cosmological singularity
}

\author{
Przemysław Małkiewicz ${ }^{\dagger}$ and Włodzimierz Piechocki ${ }^{\ddagger}$ \\ Department of Theoretical Physics \\ Soltan Institute for Nuclear Studies, \\ Hoża 69, 00-681 Warszawa, Poland; \\ †pmalk@fuw.edu.pl, ‘piech@fuw.edu.pl
}

(Dated: July 20, 2018)

\begin{abstract}
Our results concern the transition of a quantum string through the singularity of the compactified Milne (CM) space. We restrict our analysis to the string winding around the compact dimension (CD) of spacetime. The CD undergoes contraction to a point followed by re-expansion. We demonstrate that both classical and quantum dynamics of considered string are well defined. Most of presently available calculations strongly suggest that the singularity of a time dependent orbifold is useless as a model of the cosmological singularity. We believe that our results bring, to some extent, this claim into question.
\end{abstract}

PACS numbers: 98.80.Jr, 11.25.Wx, 98.80.Qc 


\section{INTRODUCTION}

In the cyclic model of the evolution of the universe the cosmic singularity (CS) plays the key role because it joins each two consecutive classical phases. We propose the following basic criterion for the choice of the model of the neighborhood of the CS: reasonable model should allow for propagation of quantum p-brane (i.e., particle, string, membrane,...) from the pre-singularity to the post-singularity epoch. If the CS constitutes an insurmountable obstacle for quantum elementary objects, the cyclic evolution cannot be realized.

One of the simplest models of the neighborhood of the CS, inspired by string/M theory, is the compactified Milne (CM) space. It was used recently [1, 2, 3] in the cyclic universe scenario.

We have already applied the above criterion for the propagation of a test particle across the CS of the CM space [4, 5]. The results we have obtained suggest that the CM space is a promising model deserving further investigation by making use of strings and membranes.

In this article we apply our criterion to the evolution of a test string in the CM space. We examine the propagation of a string in the so-called winding mode [6, 7]. It is defined to be a state in which a string is winding around the compact dimension undergoing contraction to a point followed by re-expansion. It turns out that the propagation is well defined both at classical and quantum levels.

In Sec. II we recall the classical formalism concerning the propagation of a test $p$-brane in a fixed background spacetime. Classical evolution of the string in the winding mode is presented in Sec. III. Quantization of the dynamics is carried out in Sec. IV. We conclude in Sec. V.

\section{CLASSICAL DYNAMICS OF P-BRANE}

The Polyakov action for a test $p$-brane embedded in a fixed background spacetime with metric $g_{\tilde{\mu} \tilde{\nu}}$ has the form

$$
S_{p}=-\frac{1}{2} \mu_{p} \int d^{p+1} \sigma \sqrt{-\gamma}\left(\gamma^{a b} \partial_{a} X^{\tilde{\mu}} \partial_{b} X^{\tilde{\nu}} g_{\tilde{\mu} \tilde{\nu}}-(p-1)\right)
$$

where $\mu_{p}$ is a mass per unit $p$-volume, $\left(\sigma^{a}\right) \equiv\left(\sigma^{0}, \sigma^{1}, \ldots, \sigma^{p}\right)$ are $p$-brane worldvolume coordinates, $\gamma_{a b}$ is the $p$-brane worldvolume metric, $\gamma:=\operatorname{det}\left[\gamma_{a b}\right], \quad\left(X^{\tilde{\mu}}\right) \equiv\left(X^{\mu}, \Theta\right) \equiv$ $\left(T, X^{k}, \Theta\right) \equiv\left(T, X^{1}, \ldots, X^{d-1}, \Theta\right)$ are the embedding functions of a $p$-brane, i.e. $X^{\tilde{\mu}}=$ $X^{\tilde{\mu}}\left(\sigma^{0}, \ldots, \sigma^{p}\right)$, in $d+1$ dimensional background spacetime.

It has been found [7] that the total Hamiltonian, $H_{T}$, corresponding to the action (11) is the following

$$
H_{T}=\int d^{p} \sigma \mathcal{H}_{T}, \quad \mathcal{H}_{T}:=A C+A^{i} C_{i}, \quad i=1, \ldots, p
$$

where $A=A\left(\sigma^{a}\right)$ and $A^{i}=A^{i}\left(\sigma^{a}\right)$ are any regular functions of $p$-volume coordinates,

$$
\begin{gathered}
C:=\Pi_{\tilde{\mu}} \Pi_{\tilde{\nu}} g^{\tilde{\mu} \tilde{\nu}}+\mu_{p}^{2} \operatorname{det}\left[\partial_{a} X^{\tilde{\mu}} \partial_{b} X^{\tilde{\nu}} g_{\tilde{\mu} \tilde{\nu}}\right] \approx 0, \\
C_{i}:=\partial_{i} X^{\tilde{\mu}} \Pi_{\tilde{\mu}} \approx 0,
\end{gathered}
$$

and where $\Pi_{\tilde{\mu}}$ are the canonical momenta corresponding to $X^{\tilde{\mu}}$. Equations (3) and (4) define the first-class constraints of the system. 
The Hamilton equations [7] are

$$
\dot{X}^{\tilde{\mu}} \equiv \frac{\partial X^{\tilde{\mu}}}{\partial \tau}=\left\{X^{\tilde{\mu}}, H_{T}\right\}, \quad \dot{\Pi}_{\tilde{\mu}} \equiv \frac{\partial \Pi_{\tilde{\mu}}}{\partial \tau}=\left\{\Pi_{\tilde{\mu}}, H_{T}\right\}, \quad \tau \equiv \sigma^{0},
$$

where the Poisson bracket is defined by

$$
\{\cdot, \cdot\}:=\int d^{p} \sigma\left(\frac{\partial \cdot}{\partial X^{\tilde{\mu}}} \frac{\partial \cdot}{\partial \Pi_{\tilde{\mu}}}-\frac{\partial \cdot}{\partial \prod_{\tilde{\mu}}} \frac{\partial \cdot}{\partial X^{\tilde{\mu}}}\right) .
$$

\section{CLASSICAL DYNAMICS OF STRING}

In what follows we restrict our considerations to the compactified Milne space. Its metric is defined by the line element

$$
d s^{2}=-d t^{2}+d x^{k} d x_{k}+t^{2} d \theta^{2}=\eta_{\mu \nu} d x^{\mu} d x^{\nu}+t^{2} d \theta^{2}=g_{\tilde{\mu} \tilde{\nu}} d x^{\tilde{\mu}} d x^{\tilde{\nu}},
$$

where $\eta_{\mu \nu}$ is the Minkowski metric, and $\theta$ parameterizes a circle ${ }^{1}$.

The spacetime with the metric (7) is singular at $t=0$ and it is not a coordinate choice singularity: one of its space dimensions (denoted by $\theta$ ) disappears for a moment at $t=0$ leading to the degeneracy of the metric. It is a spacelike orbifold singularity (see [4] for more details).

In the paper we analyze the dynamics of a string in the zero-mode (the lowest energy state) which is winding around the $\theta$-dimension. The string in such a state is defined by the conditions

$$
\sigma^{p}:=\theta \equiv \Theta \quad \text { and } \quad \partial_{\theta} X^{\mu}=0=\partial_{\theta} \Pi_{\mu}
$$

which lead to

$$
\frac{\partial}{\partial \theta}\left(X^{\tilde{\mu}}\right)=(0, \ldots, 0,1) \quad \text { and } \quad \frac{\partial}{\partial \tau}\left(X^{\tilde{\mu}}\right)=\left(\dot{T}, \dot{X}^{k}, 0\right) .
$$

The condition (8) reduces (3)-(6) to the form in which the canonical pair $\left(\theta, \Pi_{\theta}\right)$ does not occur [7]. Thus, a string in the winding mode is described by (3) -(6) with $\tilde{\mu}, \tilde{\nu}, \ldots$ replaced by $\mu, \nu, \ldots$ In fact, the propagation of a string 'reduces' effectively to its evolution in the Minkowski space with dimension $d$, while $d+1$ was the original dimension of spacetime [7].

The constraint equations, (3) and (4), for a string winding around the $\theta$-dimension reduce to the following form

$$
C=\Pi_{\mu}(\tau) \Pi_{\nu}(\tau) \eta^{\mu \nu}+\check{\mu}_{1}^{2} t^{2}(\tau) \approx 0, \quad C_{1}=0,
$$

where $\check{\mu}_{1} \equiv 2 \pi \mu_{1}$, and the equations of motion (5) read

$$
\dot{\Pi}_{t}(\tau)=-2 A(\tau) \check{\mu}_{1}^{2} T(\tau), \quad \dot{\Pi}_{k}(\tau)=0
$$

and

$$
\dot{T}(\tau)=-2 A(\tau) \Pi_{t}(\tau), \quad \dot{X}^{k}(\tau)=2 A(\tau) \Pi_{k}(\tau)
$$

\footnotetext{
${ }^{1}$ Orbifolding $\mathbb{S}^{1}$ to the segment $\mathbb{S}^{1} / \mathbb{Z}_{2}$ gives a model of spacetime in the form of two orbifold planes which collide and re-emerge at $t=0$. Such model of spacetime was used in [1, 2, 3], but our results do not depend on the choice of topology of the compact dimension.
} 
where $A=A(\tau)$ is any regular function.

It can be verified that in the gauge $A(\tau)=1$, the solutions are

$$
\Pi_{t}(\tau)=b_{1} \exp \left(2 \check{\mu}_{1} \tau\right)+b_{2} \exp \left(-2 \check{\mu}_{1} \tau\right), \quad \Pi_{k}(\tau)=\Pi_{0 k},
$$

where $b_{1}, b_{2}, \Pi_{0 k} \in \mathbb{R}$, and

$$
T(\tau)=a_{1} \exp \left(2 \check{\mu}_{1} \tau\right)+a_{2} \exp \left(-2 \check{\mu}_{1} \tau\right), \quad X^{k}(\tau)=X_{0}^{k}+2 \Pi_{0 k} \tau,
$$

where $a_{1}, a_{2}, X_{0}^{k} \in \mathbb{R}$.

To analyze the propagation of a string across the singularity $t=0$, we eliminate $\tau$ from (13) and (14). Making the choice of $a_{1}$ and $a_{2}$ in such a way that $a_{1} a_{2}<0$ leads to one-to-one relation between $T$ and $\tau$. For instance, one may put

$$
a_{1}=-a_{2}=\sqrt{\Pi_{0}^{k} \Pi_{0 k}} / 2 \check{\mu}_{1}
$$

that gives

$$
T(\tau)=\sqrt{\Pi_{0}^{k} \Pi_{0 k}} \sinh \left(2 \check{\mu}_{1} \tau\right) / \check{\mu}_{1}
$$

which can be rewritten as

$$
\tau=\frac{1}{2 \check{\mu}_{1}} \sinh ^{-1}\left(\frac{\check{\mu}_{1}}{\sqrt{\Pi_{0}^{k} \Pi_{0 k}}} t\right)
$$

due to $T=t$. The insertion of (17) into (14) gives

$$
X^{k}(t)=X_{0}^{k}+\frac{\Pi_{0}^{k}}{\check{\mu}_{1}} \sinh ^{-1}\left(\frac{\check{\mu}_{1}}{\sqrt{\Pi_{0}^{k} \Pi_{0 k}}} t\right) .
$$

The solution (18) is bounded and continuous at the singularity. Thus, the classical dynamics of the zero-mode winding string is well defined in the CM space.

\section{QUANTUM DYNAMICS OF STRING}

In the gauge $A=1$, the Hamiltonian of a string is

$$
H_{T}=C=\Pi_{\mu}(\tau) \Pi_{\nu}(\tau) \eta^{\mu \nu}+\check{\mu}_{1}^{2} t^{2} .
$$

The quantum Hamiltonian corresponding to (19) has the form [5]

$$
\hat{H}_{T}=\frac{\partial^{2}}{\partial t^{2}}-\frac{\partial^{2}}{\partial X^{k} \partial X_{k}}+\check{\mu}_{1}^{2} t^{2}
$$

owing to $T=t$.

According to the Dirac quantization method [8, 9] the physical states $\psi$ should first of all satisfy, due to (10) and (19), the equation

$$
\hat{H}_{T} \psi\left(t, X^{k}\right)=0 \text {. }
$$

To solve (21), we make the substitution

$$
\psi\left(t, X^{1}, \ldots, X^{d-1}\right)=F(t) G_{1}\left(X^{1}\right) G_{2}\left(X^{2}\right) \cdots G_{d-1}\left(X^{d-1}\right),
$$


which turns (21) into the following set of equations

$$
\begin{array}{cc}
\frac{d^{2} G_{k}\left(q_{k}, X_{k}\right)}{d X_{k}^{2}}+q_{k}^{2} G_{k}\left(q_{k}, X_{k}\right)=0, & k=1, \ldots, d-1, \\
\frac{d^{2} F(q, t)}{d t^{2}}+\left(\check{\mu}_{1}^{2} t^{2}+q^{2}\right) F(q, t)=0, & q^{2}:=q_{1}^{2}+\ldots+q_{d-1}^{2},
\end{array}
$$

where $q_{k}^{2}, q^{2} \in \mathbb{R}$ are the separation constants.

Two independent solutions to (23) have the form

$$
G_{1 k}\left(q_{k}, X_{k}\right)=\cos \left(q_{k} X^{k}\right), \quad G_{2 k}\left(q_{k}, X_{k}\right)=\sin \left(q_{k} X^{k}\right), \quad k=1, \ldots, d-1
$$

(there is no summation in $q_{k} X^{k}$ with respect to $k$ ).

Two independent solutions of (24) read [10]

$$
\begin{gathered}
F_{1}(q, t)=\exp \left(-i \check{\mu}_{1} t^{2} / 2\right) H\left(-\frac{\check{\mu}_{1}+i q^{2}}{2 \check{\mu}_{1}},(-1)^{1 / 4} \sqrt{\check{\mu}_{1}} t\right), \\
F_{2}(q, t)=\exp \left(-i \check{\mu}_{1} t^{2} / 2\right){ }_{1} F_{1}\left(\frac{\check{\mu}_{1}+i q^{2}}{4 \check{\mu}_{1}}, \frac{1}{2}, i \check{\mu}_{1} t^{2}\right),
\end{gathered}
$$

where $H(a, t)$ is the Hermite function and ${ }_{1} F_{1}(a, b, t)$ denotes the Kummer confluent hypergeometric function.

In what follows we present the construction of a Hilbert space, $\mathcal{H}$, of our system based on the solutions (25)-(27):

First, we intend to redefine (26) and (27) to get bounded functions on $\mathbb{R} \times\left[-t_{0}, t_{0}\right]$, where $\left[-t_{0}, t_{0}\right]$ denotes the 'time-like' neighborhood of the singularity. For fixed value of $q$ and $t \in\left[-t_{0}, t_{0}\right]$ the solutions (26) and (27) are bounded functions, as it is demonstrated by the plots of Fig. (1).
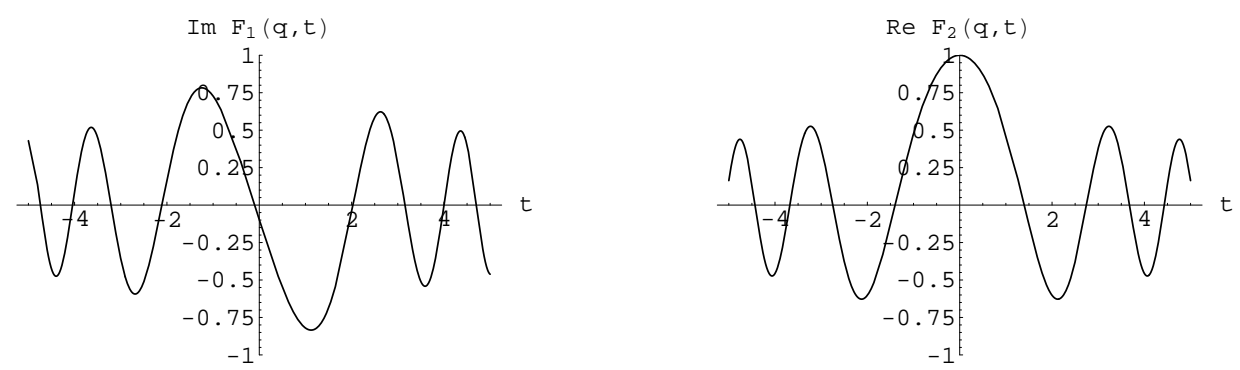

FIG. 1: Solutions as functions of $t$ in the neighborhood of the singularity $\left(\check{\mu}_{1}=1, q=1\right)$.

For $q^{2} \gg \check{\mu}_{1}^{2} t_{0}^{2}$, the solution to (24) can be approximated by

$$
F(q, t) \approx A(q) \sin (q t)+B(q) \cos (q t),
$$

where $A(q)$ and $B(q)$ are any functions. Finding bounded $A(q)$ and $B(q)$ in (28) gives bounded $F(q, t)$. They can be determined from the equations $\left(q^{2} \gg \check{\mu}^{2} t_{0}^{2}\right)$

$$
\left.F(q, t)\right|_{t=0}=B(q) \quad \text { and } \quad \partial_{t} F(q, t)_{\left.\right|_{t=0}}=q A(q) .
$$


It can be checked [10] that

$$
\begin{array}{ll}
F_{1}(q, t)_{\mid t=0}=\frac{\sqrt{\pi} 2^{\frac{-\imath q^{2}-\check{\mu}_{1}}{2 \check{\mu}_{1}}}}{\Gamma\left(\frac{3}{4}+\frac{q^{2}}{4 \check{\mu}_{1}}\right)}, & \partial_{t} F_{1}(q, t)_{\mid t=0}=\frac{(-1)^{-1 / 4} \sqrt{\pi}\left(-\imath q^{2}-\check{\mu}_{1}\right) 2^{\frac{-\imath q^{2}-\check{\mu}_{1}}{2 \check{\mu}_{1}}}}{2 \sqrt{\check{\mu}_{1}} \Gamma\left(\frac{5}{4}+2 \frac{q^{2}}{4 \check{\mu}_{1}}\right)}, \\
F_{2}(q, t)_{\left.\right|_{t=0}}=1, & \partial_{t} F_{2}(q, t)_{\mid t=0}=0 .
\end{array}
$$

It results from (30) that the solution $F_{2}(q, t)$ is a bounded function, so it does not need any redefinition. For $q^{2}$ big enough, $F_{1}(q, t)_{\left.\right|_{t=0}}$ and $\partial_{t} F_{1}(q, t)_{\left.\right|_{t=0}}$ are found to be (see Eq. $(6.1 .45)$ in [11])

$$
\left|F_{1}(q, t)_{\mid t=0}\right| \approx \sqrt[4]{\frac{\check{\mu}_{1}}{4}} \frac{\exp \left(\frac{\pi}{8 \check{\mu}_{1}} q^{2}\right)}{\sqrt{q}}, \quad\left|\partial_{t} F_{1}(q, t)_{\mid t=0}\right| \approx \sqrt[4]{\frac{\check{\mu}_{1}}{4}} \sqrt{q} \exp \left(\frac{\pi}{8 \check{\mu}_{1}} q^{2}\right) .
$$

Thus, we redefine the solution $F_{1}(q, t)$ as follows

$$
F_{1}(q, t):=\sqrt{q} \exp \left(-\frac{\pi}{8 \check{\mu}_{1}} q^{2}\right) \exp \left(-i \check{\mu}_{1} t^{2} / 2\right) H\left(-\frac{\check{\mu}_{1}+i q^{2}}{2 \check{\mu}_{1}},(-1)^{1 / 4} \sqrt{\check{\mu}_{1}} t\right) .
$$

It is clear that (32) is the solution of (24) owing to the structure of the equation. Now, one can verify that

$$
\begin{array}{ll}
\left|A_{1}(q)\right|=\sqrt[4]{\frac{\check{\mu}_{1}}{4}}, \quad & \left|B_{1}(q)\right|=\sqrt[4]{\frac{\check{\mu}_{1}}{4}}, \\
A_{2}(q)=0, & B_{2}(q)=1 .
\end{array}
$$

Therefore, we get the result that the functions $\mathbb{R} \times\left[-t_{0}, t_{0}\right] \ni(q, t) \rightarrow F_{s}(q, t) \in \mathbb{C}, \quad(s=1,2)$ are bounded.

Second, we define the following generalized functions

$$
h_{s}\left(t, X^{1}, \ldots, X^{d-1}\right):=\int_{\mathbb{R}^{d-1}} f\left(q_{1}, \ldots, q_{d-1}\right) F_{s}(q, t) \prod_{k} \exp \left(-i q_{k} X^{k}\right) d q_{1} \ldots d q_{d-1},
$$

where $q^{2}=q_{1}^{2}+\ldots q_{d-1}^{2}$, and where $f \in L^{2}\left(\mathbb{R}^{d-1}\right)$. Since $F_{s}$ are bounded, the functions $f F_{s} \in L^{2}\left(\left[-t_{0}, t_{0}\right] \times \mathbb{R}^{d-1}\right)$. Equation (34) includes (25) due to the term $\exp \left(-i q_{k} X^{k}\right)$, with $q_{k} \in \mathbb{R}$.

Finally, we notice that (34) defines the Fourier transform of $f F_{s}$. Therefore, according to the Fourier transform theory (see, e.q. [12]) the equation (34) defines the mapping

$$
L^{2}\left(\mathbb{R}^{d-1}\right) \ni f \longrightarrow h_{s} \in L^{2}\left(\left[-t_{0}, t_{0}\right] \times \mathbb{R}^{d-1}\right)=: \tilde{\mathcal{H}}
$$

Replacing $f$ by consecutive elements of an orthonormal basis in $L^{2}\left(\mathbb{R}^{d-1}\right)$ leads to an infinite countable set of vectors in $\tilde{\mathcal{H}}$. So obtained set of vectors can be rearranged into a set of independent vectors and further turned into an orthonormal basis by making use of the Gram-Schmidt procedure [13]. One can show [12] that the span of such an orthonormal basis, $\mathcal{F}$, is dense in $\tilde{\mathcal{H}}$. The completion of $\mathcal{F}$ defines the Hilbert space $\mathcal{H} \subseteq \tilde{\mathcal{H}}$.

To illustrate the above construction, let us use the Hilbert space $L^{2}\left(\mathbb{R}^{d-1}\right):=\bigotimes_{k=1}^{d-1} L_{k}^{2}(\mathbb{R})$, where $L_{1}^{2}(\mathbb{R})=L_{2}^{2}(\mathbb{R})=\ldots=L_{d-1}^{2}(\mathbb{R}) \equiv L^{2}(\mathbb{R})$. Let us take a countable infinite set of vectors $f_{n} \in L^{2}(\mathbb{R})$ defined as

$$
f_{n}(q):=\frac{1}{\sqrt{2^{n} n ! \sqrt{\pi}}} \exp \left(-q^{2} / 2\right) H_{n}(q), \quad n=0,1,2, \ldots
$$


where $H_{n}(q)$ is the Hermite polynomial. It is proved in [14] that (36) defines an orthonormal basis in $L^{2}(\mathbb{R})$. The basis (36) can be used to construct a basis in $L^{2}\left(\mathbb{R}^{d-1}\right)$. The basis is defined as the set of all vectors of the form $\bigotimes_{k=1}^{d-1} f_{n_{k}}\left(q^{k}\right) \in L^{2}\left(\mathbb{R}^{d-1}\right)$. Further steps of the procedure leading to the dense subspace $\mathcal{F}$ are the same as described in the paragraph including Eq. (35).

It is clear that (34), owing to the above construction, defines the solution to the equation $\hat{H}_{T} h_{s}=0$. It is obvious that the Hamiltonian (20) is bounded from below (and above) and self-adjoint on the Hilbert space $\mathcal{H}$.

\section{CONCLUSIONS}

Our results demonstrate that the dynamics of a test string ${ }^{2}$ winding around the compactified dimension in the zero-mode state is well defined. At the classical level there are no instabilities near the singularity (characteristic for particle dynamics) and our non-perturbative method of quantization leads to a non-singular quantum evolution of a string ${ }^{3}$.

A great challenge is examination of the dynamics of a physical string, i.e. a string that may change its state and modify the background spacetime during the evolution of the entire system. The issue of the backreaction has been intensively studied recently (see [16] for review). Calculations of the S-matrix amplitudes in time dependent singular orbifolds are plagued by infinities (see, e.g. [17, 18, 19]). However, there exist some attempts to cure the divergencies of scattering amplitudes (see, e.g. [20]). It seems to us that such results only mean that in these type of orbifolds the string perturbation theory breaks down and one should apply non-perturbative methods for analyses.

It is claimed in [21] that the pessimistic results concerning the S-matrix can be confirmed by non-perturbative analyses. One shows in [21] that, for instance, a single particle added to a time dependent singular orbifold causes the orbifold to collapse into a large black hole, which leads finally to the creation of a big-crunch that would not be followed by a bigbang $^{4}$. This result suggests that the compactified Milne space is useless as a model of the neighbourhood of the cosmological singularity in the context of the cyclic universe scenario.

It seems to us that the result of [21] should be treated with caution. It is so because it was obtained by making use of general relativity, i.e. a classical theory, which is not suitable for complete understanding of the physics of a black hole.

In our article we have proposed a new non-perturbative method of analysis of the singularity of a time dependent orbifold. We believe that it can be further applied to analyses of the propagation of a winding string in non-zero modes (excited states). It may initiate a new direction in a systematic examination of the backreaction phenomena in the context of time dependent orbifolds.

\footnotetext{
${ }^{2}$ A test string, by definition, does not change the background spacetime.

3 For the discussion of some physical aspects of propagation of winding states through the Milne space singularity we recommend [15]. One shows that the simple string cosmology based on the Milne space can be used to obtain interesting cosmologies in four dimensions.

4 See [22] for related considerations, where analyses of the bosonic and fermionic spectrum in the Milne orbifold lead the author to the conclusion that the resolution of the cosmological orbifold singularity is impossible.
} 
We are conscious that real understanding of the problem would require quantization of the entire system, including string and orbifold, but it seems to be beyond the scope of the current stage of quantum gravity.

\section{Acknowledgments}

PM would like to thank the European Network of Theoretical Astroparticle Physics ILIAS/N6 under contract number RII3-CT-2004-506222 for partial financial support. WP is grateful to Ben Craps for helpful discussion. We would like to thank the anonymous referee for the suggestion of including [15, 22] into our list of references.

[1] J. Khoury, B. A. Ovrut, N. Seiberg, P. J. Steinhardt and N. Turok, "From big crunch to big bang", Phys. Rev. D 65 (2002) 086007 [arXiv:hep-th/0108187].

[2] P. J. Steinhardt and N. Turok, "A cyclic model of the universe", Science 296 (2002) 1436 arXiv:hep-th/0111030.

[3] P. J. Steinhardt and N. Turok, "Cosmic evolution in a cyclic universe", Phys. Rev. D 65 (2002) 126003 arXiv:hep-th/0111098.

[4] P. Małkiewicz and W. Piechocki, "The simple model of big-crunch/big-bang transition", Class. Quant. Grav., 23 (2006) 2963 arXiv:gr-qc/0507077.

[5] P. Małkiewicz and W. Piechocki, "Probing the cosmological singularity with a particle", Class. Quant. Grav., 23 (2006) 7045 arXiv:gr-qc/0606091.

[6] B. Pioline and M. Berkooz, "Strings in an electric field, and the Milne universe", JCAP 0311 (2003) 007 arXiv:hep-th/0307280.

[7] N. Turok, M. Perry and P. J. Steinhardt, "M theory model of a big crunch / big bang transition", Phys. Rev. D 70 (2004) 106004 [Erratum-ibid. D 71 (2005) 029901] arXiv:hep-th/0408083].

[8] P. A. M. Dirac, Lectures on Quantum Mechanics (New York: Belfer Graduate School of Science Monographs Series, 1964).

[9] M. Henneaux and C. Teitelboim, Quantization of Gauge Systems (Princeton: Princeton University Press, 1992).

[10] S. Wolfram, The Mathematica (Software programme for computations, 2003), Version 5.2.0.

[11] M. Abramowitz and I. A. Stegun, Handbook of Mathematical Functions with Formulas, Graphs, and Mathemathical Tables (New York: John Wiley \& Sons, 1972).

[12] L. Debnath and P. Mikusiński, Introduction to Hilbert Spaces with Applications (San Diego: Academic Press, 1999).

[13] E. Prugovečki, Quantum Mechanics in Hilbert Space (New York: Academic Press, 1981).

[14] N. I. Akhiezer and I. M. Glazman, Theory of Linear Operators in Hilbert Space (New York: Dover Publications, Inc., 1993).

[15] J. G. Russo, "Cosmological string models from Milne spaces and SL(2,Z) orbifold," Mod. Phys. Lett. A 19 (2004) 421 arXiv:hep-th/0305032.

[16] B. Craps, "Big bang models in string theory", arXiv:hep-th/0605199.

[17] H. Liu, G. W. Moore and N. Seiberg, "Strings in time-dependent orbifolds," JHEP 0210 (2002) 031 arXiv:hep-th/0206182. 
[18] A. Lawrence, "On the instability of 3D null singularities," JHEP 0211 (2002) 019 arXiv:hep-th/0205288.

[19] M. Berkooz, B. Craps, D. Kutasov and G. Rajesh, "Comments on cosmological singularities in string theory," JHEP 0303 (2003) 031 arXiv:hep-th/0212215.

[20] L. Cornalba and M. S. Costa, "Time-dependent orbifolds and string cosmology," Fortsch. Phys. 52 (2004) 145 arXiv:hep-th/0310099].

[21] G. T. Horowitz and J. Polchinski, "Instability of spacelike and null orbifold singularities," Phys. Rev. D 66 (2002) 103512 arXiv:hep-th/0206228.

[22] N. A. Nekrasov, "Milne universe, tachyons, and quantum group," Surveys High Energ. Phys. 17 (2002) 115 arXiv:hep-th/0203112]. 